\title{
CHLA 2018 CONFERENCE POSTERS / ABSC CONGRÈS 2018
} AFFICHES

$\mathrm{PP}=$ Poster Presentation

\section{PP1. Engaging Learners: Interactive Ideas for Teaching Health Literacy}

Daphne Horn

Mount Sinai Hospital

According to the Public Health Agency of Canada, 55\% of working age Canadians have inadequate health literacy skills, which in turn increases to $88 \%$ for those aged 65 years or older. In order to minimize the poor health outcomes associated with low health literacy, health care providers can utilize specific tools to effectively communicate with patients and their families. Using plain language principles and incorporating the teach-back method are two tools that increase patients understanding of information, allowing them to more fully participate in their health care. As librarians' roles evolve and become more integrated in the circle of care, they become a natural fit for teaching information literacy skills to health care professionals. This poster discusses different teaching platforms that health literacy skills can be taught across, along with interactive and engaging ways to encourage participant participation and learning.

\section{PP2. Patient Library Collaborations}

Daphne Horn

Mount Sinai Hospital

To promote the newly established Patient Education Pavilion (PEP) to hospital staff and patients the Library collaborated with many hospital departments and external organizations. Using monthly national health awareness topics as a guide, and the shared goal of health promotion and patient outreach, the Library proposed activities and events to these groups. Examples of successful partnerships hosted at the PEP include music and pet therapy for Recreational Therapy Month; mustache, trivia, and prostate screening handouts for Movember; silent film screenings for National Canadian Film Day; and a Powerpoint presentation highlighting hospital volunteers for Volunteer Appreciation Week. Notwithstanding the success of these collaborative initiatives, the Library faced some institutional barriers, including insufficient library staff and volunteers, space limitations, noise issues, and uncooperative hospital employees. Despite these organizational challenges, the Library is encouraged by the positive feedback from hospital patients, families and staff.

\section{PP3. ATLAS: Advancing Teamwork for Library Accessible Services- A Communication Training Program for Library Volunteers}

Tonya Mahar, Janis Sternhill, Bruce Ballon, Shoshana Helfenbaum, Cathy Smith, Lisa Sokoloff, Faith Boucher

Baycrest Health Services 
The Baycrest Wellness Library volunteers (primarily seniors) serve predominantly geriatric patrons. While these volunteers are trained and dedicated, a needs assessment indicated that quality of service provided was inconsistent. Literature on training for this unique group is scarce and outdated, but highlights the value of senior volunteers. In response, an innovative training program, ATLAS (Advancing Teamwork for Library Accessible Services), was developed in collaboration between Library Services and Training and Simulation. ATLAS offered these volunteers the opportunity to improve their customer service skills and learn to effectively work together while assisting patrons. The library volunteers were engaged in the process of creating the training through a focus group where an appreciative inquiry approach was used to determine areas of need and set clear objectives. Based on these objectives, three experiential 90 minute workshops (Orientation, Teamwork, and Communicating with Clients) were created and delivered jointly by the Library Manager, and interprofessional and simulation educators. Evaluation was completed by all volunteers after each session, showing primarily positive responses. To evaluate the effect of the program on customer service, a 'mystery visitor' in-situ simulation approach was used 6 months post-training. Simulation Participants played roles of library patrons. They conducted a semi-scripted patron-volunteer interaction, and provided written feedback on the interaction. Ninety percent of the interactions were rated as 'a pleasant experience' by the simulation participants. With this training, volunteers are better equipped to offer customer-focused service tailored to the needs of patrons.

\title{
PP4. A Practical Toolkit for Clinicians to Locate Best Evidence
}

\author{
Sandra Kendall, Michelle Ryu, Chris Walsh \\ Mount Sinai Hospital
}

Introduction: Sinai Health System (SHS) is an internationally recognized academic health sciences centre affiliated with the University of Toronto. With more than 28,809 admissions a year, clinicians at SHS are often challenged with locating the best available evidence at the time of need. In addition to the growing number of electronic resources, long hours of clinical work and working in multiple locations prevent staff from accessing information in an efficient and timely manner. Description: The SHS Library created a toolkit that groups electronic resources available locally and remotely into tiers based on the hierarchy of evidence. The goal is to provide clinicians with an easy-to-use and practical tool to access high quality and relevant medical evidence to support quality patient care. The toolkit is also used as a valuable teaching aid by connecting clinical queries to the most relevant evidence-based resources. The toolkit guides users through the research cycle as means-test to identifying new or under-researched areas of inquiry. Outcomes: Originally published in 2008, the toolkit received positive feedback from medical students and clinical staff. The toolkit has been incorporated into the teachings of the College of Physicians and Surgeons of Ontario and Ministry of Public Health. Discussion: The authors encourage other libraries and institutions to adapt the toolkit for their users. In the future, this toolkit will be revised to tailor to the information needs of nursing and allied health staff. The toolkit will be updated to respond to changes in health information dissemination, organization, and new and emerging technologies.

\section{PP5. Expanding the Role of the Librarian as Knowledge Synthesis Methodologist: A Case Study in Teaching Rayyan}

\author{
Jill Boruff ${ }^{1}$, Martin Morris ${ }^{1}$, Nazi Torabi ${ }^{2}$ \\ ${ }^{1}$ McGill University; ${ }^{2}$ St. Michael's Hospital
}


Introduction: Health librarians assisting research teams with knowledge synthesis projects often find themselves answering questions about methodology and managing the screening process. The literature screening stage of knowledge syntheses is time consuming and difficult to track and researchers often ask for advice on ways to make this stage quicker and more efficient. This poster describes how we developed a workshop and associated online guide for Rayyan, a tool which facilitates the screening process. Methods: We researched and compared the available online screening tools. We chose to work with Rayyan for three main reasons: it is under active development (therefore, constantly improving), free to use with no restrictions, and meets most of the researchers' needs. Using feedback from researchers and librarians working on knowledge syntheses, we worked together to create a lesson plan that adapted the use of the tool to their needs. The workshop was offered without paper handouts; instead, we created an online guide to mirror the content of the lesson plan. Results and conclusions: The workshop and online guide have been popular and successful additions to the librarians' contributions to knowledge synthesis work. In 2017, four workshops reached 110 participants with positive feedback, and the subject guide has had almost 6000 visits. Rayyan is not a perfect solution, and we have developed recommendations to fill the gaps left by the tool. By sharing our experience, we hope to help other librarians looking for an open access tool that will assist researchers in the screening process.

\title{
PP6. Library Newsletters: Are They Worth it?
}

\author{
Me-Linh Lee, Laurie Blanchard \\ University of Manitoba
}

Introduction: Academic and hospital health libraries serve a diverse set of users, some of whom may be distributed across a wide geographic range and never physically set foot in the library. it is essential that libraries find effective ways to communicate about their programs, resources, and services online. At Institution $\mathrm{X}$ we send out a newsletter to subscribers once a month highlighting, among other things, upcoming training sessions, new resources, and relevant or interesting items. However, with the wide variety of other communication tools now available (social media, websites, listservs) is a newsletter still the best way to communicate with our users? Methods: An online survey using Survey Monkey will be distributed to over 1,000 subscribers to determine their satisfaction with the newsletter. Subscribers include students, residents, professional health care workers, faculty, staff, and researchers from a large academic institution and its affiliated healthcare hospitals and health centres. Usage statistics generated by Mail Chimp and WordPress will also be analyzed. Results: The survey will be distributed in early 2018 and results presented at the conference. Discussion: The majority of Canadian health facility libraries use newsletters to communicate with their users, and the amount of staff time dedicated to creating, maintaining, and distributing them is not insignificant. In times of fiscal restraint across libraries, is that effort sustainable? The results of this study are intended to help guide the decision-making process at one institution, but can hopefully be applied to any library that produces a newsletter.

\section{PP7. Health Professionals' Awareness of Predatory Journals}

\author{
Maureen Babb, Orvie Dingwall \\ University of Manitoba
}

Introduction: Predatory journals have become a serious problem in recent years; they threaten to compromise the integrity of medical literature by introducing poor quality material into the academic 
record. Librarians and editors have been at the forefront of raising awareness about such journals, but have primarily focused on advising academics to avoid publishing in predatory venues. Healthcare professionals engage with the medical literature regularly, using it in their practice, to inform their own research, and to develop policy. As such, it is important that they be aware of the issues posed by predatory journals. This study assesses the level of awareness of health professionals regarding predatory journals, and explores the effectiveness of education sessions in raising that awareness. Methods: During a continuing education session delivered to health professionals, attendees rated their familiarity with predatory journals. In response to their low levels of familiarity, a continuing education session was developed, to assist health professionals in becoming aware of the existence and perils of predatory journals. Session attendees were surveyed on their prior familiarity with such journals, and the effectiveness of the education session. Results: Participants expressed little to no prior awareness of predatory journals. Education sessions were an effective means of raising their level of awareness. Discussion: While healthcare professionals require awareness of predatory journals to accurately assess the information they use on a daily basis, their level of awareness tended to be very low. Education sessions appear to be an effective means of raising healthcare professional awareness of predatory journals.

\title{
PP8. Non-Traditional Library Instruction: St. Michael's Hospital Health Sciences Library (HSL) Excel Training Program
}

\author{
Alissa Epworth \\ St. Michael's Hospital
}

Introduction: In 2014, a HSL user survey identified the need for Excel training which was not being offered by any other hospital department. Though outside the traditional scope of education provided by a medical library, it was determined that the introduction of Excel courses met our mandate to enable the hospital community to access existing knowledge and create new knowledge. Description: We piloted a one-hour hands-on workshop targeted to all potential library patrons, including staff, students and volunteers at the hospital. The well attended workshops identified challenges with variances in levels of previous knowledge and learning. To address this, the content was divided and expanded to include both Basic and Intermediate Excel workshops. Continued demand, measured through workshop evaluation responses, resulted in the further addition of an Excel Pivot Tables course. Outcomes: Excel is the most attended workshop in our portfolio, making up 51\% of total attendance across core offerings. By offering non-traditional workshops, such as Excel, HSL workshop attendance has continued to increase, and is up $700 \%$ from 2011. Discussion: By diversifying our workshop portfolio and responding to user demand, we have substantially increased the number of visitors to the library. In addition we are reaching a user group that is outside of our core clientele (i.e. administrative, clerical, non-clinical, non-research professionals). This has proved to be an effective means of organically growing our user base and interest in the Health Sciences Library in general.

\section{PP9. Student Success: Undergraduate Engagement on a Systematic Review}

\author{
Lisa Demczuk, Kendra Rieger, Sochimaobi Nweze \\ University of Manitoba
}


Systematic review teams frequently engage student research assistants (RA). Undergraduate RAs, with less education and experience, bring energy and enthusiasm, but also the need to inspire further research interest and skill development. We report on the approach used to achieve a successful student experience and systematic review. A nursing research intern, supported by an Undergraduate Research Award, was engaged on a systematic review. A primary goal of the internship was developing research skills and experience. The review's principal investigator (PI) and librarian took a practical, outcomes-focused approach to the student's orientation, task assignment, and expected deliverables. The PI was responsible for mentorship and organizing the student's overall experience, including training, assignment of review tasks, and work supervision. The librarian worked closely with the student, providing instruction, consultation, and feedback on the tasks. Development activities included instruction on systematic review procedures and database/grey literature searching, guidance for keyword and subject heading identification, and training for reference management and article screening. Student deliverables included developing a search terms table, testing librarian-developed searches for key articles, grey literature searching, initial title/abstract screening, organization of RefWorks and EndNote libraries, and, full-text article retrieval including document delivery requests. The review successfully moved through the initial stages with an undergraduate RA and is nearing completion. Undergraduate students can be successful RAs for a systematic review. Review team leads and librarians can collaborate to provide effective learning and skill development experiences for research interns while maintaining rigour in the review process.

\section{PP10. A Concept Map to Support Question Formulation in Evidence-Based Practice (EBP) Instruction in a Nursing Curriculum}

Francesca Frati

McGill University

Introduction: The PICO framework facilitates question formulation and improves the success of searches by identifying the Problem/Patient, Intervention, Comparison and Outcome. It helps distinguish multiple questions within complex scenarios and identify key concepts for each. However, PICO works best for effectiveness questions, while nursing and other specialties often encounter other types of questions such as those about experience or meaning, and implementation. Several frameworks have been developed to address qualitative and other questions, however the thought process that accompanies question formulation is more important that the choice of framework. Description: A concept map illustrating how PICO relates to type of question and best evidence was previously developed and used effectively to teach hematology residents, undergraduate medicine students, and hospital based occupational therapists and nurses. To better reflect the nature of nursing questions, a modification to this map allows for the use of any or no framework and focuses on identifying individual questions and related key concepts as the essential first step in the process of question formulation. A further modification has been the inclusion of implementation questions, and higher levels of evidence based on the $6 \mathrm{~S}$ evidence pyramid to reinforce the importance of seeking out pre-appraised, synthesized evidence when available. The updated map is interactive, linking to an online 6S evidence pyramid subject guide. Results/Discussion: The updated concept map helps illustrate how key concepts relate to type of question and best evidence when addressing questions that do not lend themselves to the PICO framework during EBP instruction. 


\title{
PP11. A New Role in Systematic Review Support: Collaborations between Health and Social Science Librarians
}

\author{
Jackie Stapleton, Sarah Brown \\ University of Waterloo
}

Introduction: The growing nature of interdisciplinary research and collaborations between researchers across academic faculties are expanding the role of systematic review support in the academic library. In Fall 2017, a health librarian and social sciences librarian teamed up to develop a systematic review workshop targeted towards social science researchers. Description: Drawing on experience from previous systematic review collaborations, it became clear that there were misconceptions and an unfamiliarity with systematic review methods among social science researchers. To educate researchers and gauge the need for systematic review instruction, a health and social sciences librarian designed a workshop intended as a high-level introduction to systematic review methods. The workshop focuses on techniques specific to social science research questions and information sources. Outcomes: Although initially unsure as to the level of interest in the workshop, registration quickly exceeded capacity and additional workshops were scheduled. Workshop data and participant feedback will be collected and used for evaluation purposes. In particular, data will be collected to determine which academic departments attended the workshop, the relevance of the content and how the participants intend to use this knowledge. Discussion: The collaboration between the health and social science librarians resulted in a mutual exchange of skills and subject expertise. As the need for systematic review support continues to expand beyond the health sciences, building a community of practice among academic librarians in a variety of disciplines will be vital.

\section{PP12. Stretching PICO: Implications for Database Searching and Perceived Searching Confidence}

Jody Nelson, Lisa Shamchuk

MacEwan University

Introduction: Library Technician (LT) students develop knowledge and expertise in translating diverse patron reference questions into effective search strategies. Traditionally they are taught generic concept mapping for identification of searchable components. This poster explores how PICO could be modified and applied, outside of the clinical context, as a novel teaching approach to structuring search strategies and promoting searching confidence in information literacy instruction across disciplines. This study examines the effectiveness of PICO vs generic concept mapping as searching strategies for first year LT students, also considering student preference, and any differences in perceived searching confidence. Methods: Classroom instructor and librarian collaboratively facilitated guided searching activities, wherein students employed both PICO and generic strategies, submitting their search results and personal reflections via an online form. Responses were analyzed for differences in the quality of search results, in students' indication of preference, and in the students' perceived confidence scores. Results: Initial analysis of search results revealed comparable recall between the two strategies but greater precision with PICO searches. Students commented on the greater effectiveness of PICO for structuring a focussed search, yet no clear preference was noted. Self-rated searching confidence results were not significantly different between the two strategies. Discussion: Though no strong preference was indicated, both strategies appeared useful to students depending on the context of the question. The instructor will continue to teach both generic concept mapping and PICO, thus equipping these undergraduate students for the various information seeking requests they will encounter, as either students or as library professionals. 


\title{
PP13. Rants, Reflection and Revisions: Faculty-Librarian Collaboration to Improve Senior BScN Critical Analysis Learning
}

\author{
Jody Nelson, Elizabeth White-MacDonald \\ MacEwan University
}

Introduction: Nursing faculty collaborated with the nursing librarian for strategies to promote in-depth analysis and evaluation of nursing knowledge for fourth year $\mathrm{BScN}$ students. With librarian support, faculty designed a discourse analysis assignment, requiring students to apply critical textual analysis through a nursing framework, and integrate evidentiary support. Resultant assignments tended to lack the requisite depth of analysis or desired integration of nursing theory and knowledge. Collectively reflecting on the challenges, the faculty-librarian collaboration moved to a partnership which saw the development of a novel teaching approach. The revised partnership focused on strategies related to class structure and process, as well as the evaluative strategies intended to enhance student learning. Description: In Fall 2017, the faculty-librarian team facilitated critical textual analysis classes and scaffolded learning, which afforded students low-stakes opportunities to apply concepts in a supportive environment prior to completing the evaluative assignment. This quality improvement project includes an analysis of student reflective and evaluative assignments. We will reflect on how revisions influenced depth of analysis and effectiveness of integration of evidence, theory and nursing knowledge with this cohort. Outcomes: Overall, student assignments demonstrated deeper textual analysis and more effective application of discourse analysis concepts. Nursing knowledge was not integrated to the degree that we had anticipated. Discussion: We will share insights from this small-scale quality improvement project and address how our novel teaching design served to promote student learning in this cohort. Additionally, we will discuss the value of librarian-faculty collaboration in supporting student success, hopefully inspiring other faculty-librarian partnerships.

\section{PP14. Patient Information Aid: An Innovative Educational Program to Improve Outcomes of Online Consumer Health Information}

\author{
Pierre Pluye ${ }^{1}$, Reem El Sherif ${ }^{1}$, Vera Granikov ${ }^{1}$, Malik Altakrori ${ }^{1}$, Fabio Balli ${ }^{2}$, Maria Cristiane \\ Barbosa Galvao ${ }^{3}$, Bernard Burnand ${ }^{4}$, Francesca Frati ${ }^{1}$, France Légaré ${ }^{5}$, Mathieu Bujold ${ }^{1}$, Roland \\ Grad $^{1}$ \\ ${ }^{1}$ McGill University; ${ }^{2}$ Concordia University; ${ }^{3}$ University of Sao Paulo; ${ }^{4}$ Lausanne University Hospital; \\ ${ }^{5}$ Université Laval;
}

Introduction: The volume of online consumer health information (OCHI) is ever-growing. However, OCHI quality varies, which may lead to positive and negative health outcomes. Based on results of a systematic review and a qualitative study, we designed an educational program the Patient Information Aid (PIA). The PIA website is aimed to facilitate information seeking, enable positive outcomes of OCHI, and reduce negative outcomes. Description: A systematic mixed studies review uncovered positive and negative outcomes of $\mathrm{OCHI}$, and a qualitative study identified strategies to reduce negative outcomes. As a result, we proposed a conceptual framework of OCHI outcomes, and suggested that negative outcomes may be reduced when consumers are supported in searching, assessing and discussing OCHI with their social networks and health professionals. These results led to design PIA. Before people start searching for information, PIA provides searching tips and links to reliable sources, e.g., the Medical Library Association's list of websites. During search, PIA helps them assess sources using a friendly checklist based on Health On the Net (HON) standards. After finding potentially relevant and reliable information, PIA encourages them to 'save' and 'share' with relatives and health professionals when needed. Outcomes: The beta-version of the PIA website will be presented at the conference. PIA users will rate 
and comment on OCHI they find using the validated Information Assessment Method. Discussion: PIA constitutes an innovative educational program. PIA is based on research results and addresses three searching stages (before, during, after) in an iterative manner.

\title{
PP15. Collaborative Monitoring and Filtering of Patient-Oriented Research Publications: A Protocol for a Mixed Methods Study
}

\author{
Vera Granikov, France Bouthillier, David Li Tang, Pierre Pluye \\ McGill University
}

Introduction: Keeping up-to-date is intrinsic to research, but is challenging due to information overload, time constraints, and insufficient evaluation skills. This is particularly true for Patient-Oriented Research (POR), which is meant to engage researchers, patients, clinicians, and decision-makers (i.e., POR stakeholders), with diverse research experience and skills. Collaboration may provide a solution. Our objective is to explore collaborative monitoring and filtering from the perspective of POR stakeholders. Methods: The project is based on an innovative collaborative research trends monitoring system and will follow an explanatory sequential mixed methods design. A quantitative longitudinal study with system users (POR stakeholders): data will be collected automatically by the system (e.g., number of abstracts read and rated). Descriptive statistics summarizing system usage data will inform a qualitative multiple case study with a purposeful maximum variation sample of participants, until reaching data saturation. Data collection will include semi-structured interviews about participants' experience with collaborative monitoring and filtering, and will be complemented by researcher diary and correspondence with system users. Data will be analyzed using inductive and deductive thematic analysis. Quantitative data will inform qualitative data collection; quantitative and qualitative results will be compared. Results: Two POR communities are using the system; two others are in development. Discussion: The project will advance knowledge regarding the processes and outcomes of collaborative monitoring and filtering of POR trends, important to those providing monitoring services or studying collaborative information behaviour. The findings will contribute to our understanding of current awareness practices and will inform future system design.

\section{PP16. Quiet Down at the Library: Promoting the Library as a Place/Source of Wellness}

\section{Sandy Iverson, Bridget Morant}

St. Michael's Hospital

Introduction: St Michael's Hospital library partnered with their corporate health and safety department to offer a series of events to help hospital staff and students manage stress, and focus on wellness while simultaneously promoting the library space and services. Health and wellness programming is a popular trend in public libraries, but seldom offered in hospital libraries despite health and wellness being top of mind for health care staff. The aim of this program was to promote the library as a place/source of wellness. Framing the library as a source of wellness aligned the library with a pre-existing concern of health care staff and the library enjoyed increased engagement with its clients as a result. Description: Monthly workshops or activities addressing stress management and wellness were held in the library over a five month period. Sessions were facilitated by clinical staff and/or library staff. Sessions included crafts, games, therapy dogs, aromatherapy, meditation and yoga. Events were promoted throughout the hospital and on social media. Outcomes: Most sessions were over enrolled, indicating a high level of 
interest in these topics. Attendance records and brief evaluations were collected and analyzed. All sessions were extremely well received. Discussion: The library benefited from this promotional effort by enhancing their image as a place of active engagement in the hospital community and as being sensitive to staff and student needs beyond providing traditional library services. Over 300 people attended the most popular session, which made it the most popular event this library has ever held.

\title{
PP17. An International Survey of Grey Literature Searching Practices in Horizon Scanning
}

\author{
Monika Mierzwinski-Urban, Kelly Farrah \\ CADTH
}

Introduction: Information on new and emerging technologies is often not available in traditional bibliographic databases such as PubMed. As such, there is an increased reliance on grey literature in horizon scanning on health technologies. The objective of this survey was to gain an understanding of current practices in searching for grey literature among producers of horizon scanning reports internationally. Methods: Members of the EuroScan International Network and other international agencies that produce horizon scanning reports were invited to complete an online survey. The survey included questions about whether and how grey literature is searched, which types of grey literature are searched, and how the searches are documented. Results: Sixteen horizon scanning agencies completed the survey. All but one reported always or frequently conducting a grey literature search for horizon scanning reports. The categories of grey literature most frequently reported as always searched were: regulatory agencies (95\%), clinical trial registries (88\%), horizon scans/health technologies assessments $(88 \%)$, and manufacturer information $(81 \%)$. Half of the agencies reported using a checklist to guide the grey literature search. Half of the agencies documented the grey literature search in the final report. The time spent on conducting a grey literature search varied from two hours to ten days. Discussion: There was high agreement that horizon scanning agencies are conducting grey literature searches and which types of sources were the most frequently searched. However, there was wide variation in how the grey literature searches are conducted and documented and how much time is spent on the searches.

\section{PP18. Standards for Literature Searching: Validation of a Proposed Model for Search Methods}

\author{
Brooke Ballantyne Scott ${ }^{1}$, Ashley Farre $\|^{2}$, Jackie MacDonald ${ }^{3}$, Susan Baer ${ }^{4}$, Marcus Vaska ${ }^{5}$, Pat \\ Lee $^{6}$, Danielle Rabb \\ ${ }^{1}$ Fraser Health; ${ }^{2}$ Univeristy Health Network; ${ }^{3}$ Dalhousie University; ${ }^{4}$ Saskatchewan Health Authority; \\ ${ }^{5}$ Alberta Health Services; ${ }^{6}$ Halifax, NS; ${ }^{7} \mathrm{CADTH}$
}

Objectives: Through three years of iterative literature review, research and development, a pan-Canadian group of librarians have outlined model search methods for handling each of five progressive levels of search complexity, supported by a lexicon glossary for mediated literature searching collected from the literature. This online questionnaire seeks to compare what mediated searchers 'should do' with what they actually do. Methods: As Part 2 of a multi-methods research study, our online questionnaire asked mediated searchers $(n=104)$ to choose one of 13 exemplary searches and describe how they handled a recent similar search. Responses were analyzed by search level in subgroups defined by mediated searchers' years of experience searching, and number of searches completed weekly. Data were then represented graphically to illustrate congruency within and between groups, and between questionnaire respondents and the model. Incongruities were flagged and addressed through reflexivity and 
consultation. Results: The results of our online questionnaire were analysed and any incongruities between our placement and inclusion of certain steps within the search stages and the questionnaire responses were debated as a group. Preliminary questionnaire analysis suggests that mediated searchers may not always tailor search methods to search complexity, possibly 'over-searching' or 'under-searching' as a result. Inconsistencies within and between subgroups as defined by years of experience suggest inconsistencies in search education/training. Some respondents indicated uncertainty with search terms. Conclusions: This questionnaire study highlights inconsistencies in approaches used by mediated search professionals. Although client satisfaction with mediated search services has been reported in research literature, professional searchers have worked.

\title{
PP19. Les bibliothécaires pourraient-ils appuyer les chercheurs?
}

\author{
Jairo Buitrago Ciro
}

Université d'Ottawa

Introduction : L'anglais est la langue de la diffusion scientifique, bien qu'un pourcentage faible de la population mondiale soit anglophone. Comment les chercheurs non anglophones accèdent-ils à la littérature? La traductique joue-t-elle quel rôle? Comment les bibliothécaires peuvent-ils les appuyer? On présente une enquête menée auprès de chercheurs en santé en Colombie pour déterminer si la bibliothèque peut les appuyer. Bien que cette enquête vise les colombiens, les résultats sont pertinents pour le Canada, qui a des chercheurs francophones ainsi qu'un nombre croissant de chercheurs internationaux. Méthodologie: Nous avons sondé des chercheurs hispanophones qui travaillent en sciences de la santé à une université colombienne pour déterminer pourquoi, quand, comment et combien de fois ils utilisent la traductique pour accéder à la littérature, ainsi que le type de soutien dont ils ont besoin. Résultats: La grande majorité des 46 répondants utilise amplement la traductique pour accéder à la littérature. Cependant, ils expriment des inquiétudes quant à la qualité et ils ont des connaissances limitées sur la façon d'optimiser leurs interactions avec ces outils. Une écrasante majorité aimerait recevoir une formation pour mieux utiliser la traductique. Exposé: En tant qu'experts de la culture numérique, les bibliothécaires sont bien placés pour appuyer les chercheurs en leur offrant une formation en la littératie de la traductique. Maintenant que le besoin a été identifié, l'étape suivante consistera à concevoir une formation.

\section{PP20. The Residential School Experience Through the Eyes of Indigenous Children's Authors}

\author{
Andrea Quaiattini ${ }^{1}$, Maria Tan ${ }^{2}$, Sandy Campbell ${ }^{2}$ \\ ${ }^{1}$ McGill University; ${ }^{2}$ University of Alberta
}

Introduction: Residential school experiences and their legacies underpin many health and public health challenges faced by Canada's Indigenous peoples. This poster presents the findings of Part 1 of a two-part study, which addresses the residential school experiences and the reconciliation process as they appear in children's books (PreK-12) authored by Canadian Indigenous people. Part 1 includes content related to the residential school experience and its legacy. Books selected for this study were reviewed by one or more of the researchers who identified and came to consensus on themes related to the Indigenous residential school experience. We present examples from books that illustrate themes identified in the research. Methods: The Amazon 100 Bestseller Canadian Indigenous Story Books list was sampled over a sevenweek period; additional titles were gathered from publishers, academic, and public library book lists. A 
list of books selected for the project was organized according to the residential school themes. Results $\boldsymbol{\&}$ Discussion: Participants at this session will gain an appreciation for the breadth of Indigenous children's publications related to residential schools in Canada. They will also have access to a list of fictional works on this topic that were created by Canadian Indigenous authors and/or illustrators. The list can be used by diverse groups looking for stories that promote awareness, discussion, and understanding of residential schools and their legacy.

\title{
PP21. Librarian as Course Collaborator: An Embedded Model of Information Literacy Instruction for First-Year Pharmacy Students
}

\author{
Caitlin Carter, Sherilyn Houle \\ University of Waterloo
}

Introduction: Over the past decade, there has been an increase in the number of academic librarians' pursuing embedded approaches to teaching in specific courses, as opposed to teaching 'one-shot' instructional sessions. The published literature includes examples from the University of Arizona's Health Sciences Library, Duke University's Medical Center Library, and many others. Becoming 'embedded' into a course involves close collaboration with faculty and includes duties like content development, lecturing, assignment creation and grading, as well as maintaining an online presence via a course management system. This poster outlines a librarian's role in collaborating with a faculty member on delivering IL instruction in a first-year pharmacy course. Description: The School of Pharmacy undergraduate curriculum includes a required, first-year course called 'Introduction to Drug Information Fundamentals.' The librarian develops and delivers 4 lectures on topics in information literacy and evidence-based medicine, in addition to creating and grading a PubMed searching quiz and questions for the midterm. The faculty member instructs on interpreting biostatistics and performing critical appraisal, in addition to developing and grading course assignments. Outcomes: Feedback was gathered from students using Top Hat and Evaluate and was mostly positive. Performance on course requirements illustrates the level of student success in the course. Discussion: Becoming embedded into a course is a very enriching experience for a librarian. Not only does it provide librarians with the opportunity to showcase their IL skills, but it facilitates relationship building with faculty and students, as well as a better understanding of student information needs.

\section{PP22. Telling Canadian Research Data Management (RDM) Stories in the Health Sciences}

\author{
Lindsey Sikora ${ }^{1}$, Helen Brown ${ }^{2}$, Marlene Dorgan ${ }^{3}$, Martin Morris ${ }^{4}$, Alexandra Cooper ${ }^{5}$, Leslie \\ Barnes $^{6}$, Melissa Cheung ${ }^{1}$, Ursula Ellis ${ }^{2}$, K-Lee Fraser ${ }^{3}$, Katherine Miller ${ }^{2}$ \\ ${ }^{1}$ University of Ottawa; ${ }^{2}$ University of British Columbia; ${ }^{3}$ University of Alberta; ${ }^{4}$ McGill University; \\ ${ }^{5}$ Queen's University; ${ }^{6}$ University of Toronto;
}

Introduction: Canadian funding agencies' anticipated requirements around data sharing, data preservation, and the creation of data management plans (DMPs) have prompted the development of a national research data management strategy. Academic librarians are contributing to this conversation by developing new services and infrastructure that help researchers manage their data throughout the project life cycle. To better understand how academic libraries can best further the national RDM agenda and support researchers, librarians at 9 Canadian universities have collaborated to survey researchers in medicine and health sciences regarding their RDM needs and practices, generating both institutional and 
national results. Methods: To examine the RDM practices and needs of health sciences and medical researchers, representatives from each library distributed a standard, multi-institutional survey. The survey results will generate a richer understanding of disciplinary practices and the state of RDM both locally and nationally. Results: Results will be available by May 2018. Discussion: The present study focuses on the RDM practices and future needs of health and medical researchers and expands on two previous surveys: one of the social sciences and humanities, and another of the physical sciences and engineering. This national partnership will help to inform libraries, researchers, and other stakeholders across Canada on the national, provincial, and local level to help them build a cohesive and reflective data service.

\title{
PP23. Collaborative Assessment: Developing a Library Assessment Framework for a Distributed Medical Program Libraries in British Columbia
}

\author{
Rebecca Raworth ${ }^{1}$, Vanessa Kitchen ${ }^{2}$, Mathew Vis-Dunbar ${ }^{3}$, Trina Fyfe ${ }^{4}$, Erin Menzies ${ }^{3}$ \\ ${ }^{1}$ University of Victoria; ${ }^{2}$ University of British Columbia; ${ }^{3}$ University of British Columbia Okanagan; \\ ${ }^{4}$ University of Northern British Columbia
}

Introduction: The objective of this project was to develop a thoughtful but simple assessment framework for use at both university and hospital libraries supporting a distributed MD undergraduate program across a large geographic space. Currently, there are no best practices for assessing library services at distributed medical programs. Description: By determining the unique needs of a distributed medical program and its highly varied libraries (e.g. distributed, with several hubs, managed through various university and health authority libraries) a pilot assessment framework was developed to assess broad service levels and the quality of research support and information provision for MD undergraduate students. Outcomes: Many aspects of library services will be considered including pedagogical instruction, research and publication support, including scoping and systematic review support, and collection development streamlining. Discussion: We aim to create and share a best practices document for assessing similar distributed MD Undergraduate library programs and services.

\section{PP24. How Can 'Pain' be Found? Preliminary Analysis of Searching for Pain Topics for Systematic Reviews}

\author{
Melanie Anderson \\ University Health Network
}

Introduction: Pain can be a complicated topic to perform a systematic search for, with many subject headings and keywords to consider. In order to gain insight into how to effectively find studies addressing pain for systematic reviews, this is a preliminary analysis of how systematic reviewers are currently searching for pain topics, and how that compares to how articles about pain topics are indexed in medical databases. Methods: Systematic reviews published in 2017 under the Pain, Palliative and Supportive Care group in the OVID Cochrane Database of Systematic Reviews formed the basis for this analysis. The database selection and the use of subject headings and keywords relating to pain pulled from the search strategies as well as the indexing and keywords from the records for the articles selected for inclusion were examined. Results: In these reviews, primarily Medline, Embase, and Cochrane Central were searched, although some included between one and seven additional databases. The pain related search terms varied significantly both between and within specific pain topics. The articles 
selected for inclusion in these systematic reviews share some subject headings across pain topics, however some headings or keywords are unique to a specific pain topic. Discussion: In this preliminary analysis, potential patterns are identified in the search strategies of recent pain topic Cochrane systematic reviews as well as in the bibliographic records of papers selected for inclusion in these reviews.

\title{
PP25. Evaluating the Impact of an Information Specialist as a Knowledge Broker
}

\author{
Sheila Tucker \\ CADTH
}

Publicly funded organizations which produce information are increasingly expected to demonstrate their value through evaluation of the impact of their services. Within the continuum of information services, there is now an additional component of evaluation and impact assessment. This involves the evaluation of the impact that information providers and knowledge translation specialists have on identified stakeholders and decision-making forums. The role of the knowledge broker is essential in this process, as they provide a two-way link between information producers and users. Their activities include: information needs assessment; facilitating the development and delivery of information and supporting the evaluation of these methods; assessing the impact of information services; and identifying innovations in the transfer of information based upon the evaluation (both formal and informal) of outcomes. This poster presentation will discuss the role of an information specialist as a knowledge broker in the process of building organizational capacity to evaluate the impact of processes and outcomes. Specific examples of information and knowledge exchange services which have influenced policy and practice in the health sector will be discussed with a focus on the role of the information specialist in this process.

\section{PP26. In Search of Nursing's History}

\section{Aleteia Greenwood, Katherine Miller University of British Columbia}

Introduction: In Search of Nursing's History is a half-day symposium sharing collections and stories about partnerships between the School of Nursing at UBC and units at UBC Library and Archives. Librarians and archivists from several units will highlight their collaborations with the School of Nursing. Description: This poster will present to the CHLA community one example of a partnership between the School of Nursing and the library. March 8th, 2018 Woodward Library will host, In Search of Nursing's History a symposium highlighting the partnerships between UBC Library (Archives, cIRcle, Special Collections, and Woodward Library) and the School of Nursing and its impact on scholars. The 2018 Nursing History Symposium highlights new and existing primarily open access resources in nursing history on campus and beyond. Outcomes: This symposium will be an opportunity to shine a spotlight on the work of archives and the library to further the work of researchers in nursing history. It is also an opportunity to welcome scholars into the historic space for a walkabout tour of exhibits featuring some of the unique and valuable nursing collections. Discussion: Some reflections on the lessons learned from this event. Opportunities for further partnerships and promotion of library collections and services. 
PP27. Collaboration by the numbers: How many librarians does it take to teach a medical student?

Jessica Hanley ${ }^{1}$, Kaitlin Fuller ${ }^{1}$, Daphne Horn ${ }^{2}$

${ }^{1}$ University of Toronto; ${ }^{2}$ Mount Sinai Hospital

The Health Science Research (HSR) Working Group is a collaboration of 16 academic and hospital librarians from 6 different institutions whose mandate is to support information literacy instruction to students enrolled in the MD program at a large research university. The group works in coordination with the HSR curriculum committee through the university's liaison librarians. To meet newly created curricular objectives, the working group created three new online modules, worksheets, an evaluation rubric, a tutor's marking guide, and an in-person instructional session. The instructional session was designed for 2nd year medical students, needed to be taught at the hospital academy sites, and needed to follow the programs flipped classroom model. The group met throughout the year to collaborate on content development, lesson plans, evaluations, and numerous logistics. On October 4th 21 librarians, and 3 graduate library school students successfully coordinated and taught a 2 hour class at the same time, to 268 students, across 7 separate locations, throughout the metropolitan area. This poster will focus on how the collaboration came together, and will discuss what worked and lessons learned when collaborating with a group of such significant size. 